Cahiers $d u$ MONDE RUSSE

\section{Cahiers du monde russe}

Russie - Empire russe - Union soviétique et États indépendants

$53 / 4 \mid 2012$

Varia

\title{
Ivan Foletti, Da Bisanzio alla Santa Russia| N. P. Kondakov, Iconographie de la Mère de Dieu
}

\section{Olga Medvedkova}

\section{OpenEdition \\ Journals}

Édition électronique

URL : http://journals.openedition.org/monderusse/7802

DOI : $10.4000 /$ monderusse. 7802

ISSN : $1777-5388$

Éditeur

Éditions de l'EHESS

\section{Édition imprimée}

Date de publication : 15 décembre 2012

ISSN : $1252-6576$

Référence électronique

Olga Medvedkova, «Ivan Foletti, Da Bisanzio alla Santa Russia | N. P. Kondakov, Iconographie de la Mère de Dieu », Cahiers du monde russe [En ligne], 53/4 | 2012, mis en ligne le 02 décembre 2013, Consulté le 24 septembre 2020. URL : http://journals.openedition.org/monderusse/7802 ; DOI : https://doi.org/10.4000/monderusse.7802

Ce document a été généré automatiquement le 24 septembre 2020.

(c) École des hautes études en sciences sociales 


\title{
Ivan Foletti, Da Bisanzio alla Santa Russia | N. P. Kondakov, Iconographie de la Mère de Dieu
}

\author{
Olga Medvedkova
}

\section{RÉFÉRENCE}

Ivan FolETTI, Da Bisanzio alla Santa Russia. Nikodim Kondakov (1844-1925) e la nascita della storia dell'arte in Russie. Roma : Viella, 2011, 290 p.

N. P. KONDAKOV, Iconographie de la Mère de Dieu. Vol. III. Introduction et édition d'Ivan Foletti. Rome : Lipa, 2011, 609 p.

1 Ce livre de Foletti, issu d'une thèse de doctorat dirigée par le professeur Serena Romano à l'université de Lausanne, vient de paraitre dans la collection «Études lausannoises d'histoire de l'art ». Le professeur Romano, qui dirige cette collection aux éditions de Viella à Rome, a également rédigé l'introduction de cette synthèse consacrée à l'historien de l'art russe Nikodim Kondakov dont l'importance est indéniable pour les études de l'art byzantin et russe médiéval, ainsi que, de manière plus large, pour les études médiévales.

2 Le livre d'Ivan Foletti se divise en trois parties. Dans la première, l'auteur retrace les principales étapes de la biographie de Kondakov (Cenni biografici) en s'appuyant sur les Mémoires de Kondakov (Vospominanija i dumy), publiés en exil en 1927 et réédités en 2004 à Moscou avec une introduction d'Irina Kyzlasova, spécialiste de son œuvre. Ivan Foletti souligne l'importance des origines modestes de Kondakov - fils de l'intendant des princes Trubeckoj, pour qui l'université, la science et les études byzantines devinrent un outil de promotion sociale. Sont retracées ensuite les années d'études, de voyages, d'enseignement à l'université d'Odessa et celle de Saint-Pétersbourg, le travail de conservateur à l'Ermitage et les publications qui se succèdent à un rythme soutenu. 
La révolution de 1917 fut vécue par Kondakov comme un drame personnel. Après un séjour à Odessa et à Jalta, où il possédait une datcha, il quitta la Russie en février 1920, à l'âge de 76 ans. Il passa quelque temps à Constantinople et à Sophia, puis fut accueilli à Prague: ces années pragoises sont d'autant plus importantes pour nous, souligne Foletti, que Kondakov y fonda une véritable école.

Dans la deuxième partie de l'ouvrage, Foletti analyse l'étude par Kondakov de l'icône russe (Nikodim Kondakov e l'icona). L'icône byzantine, grecque et russe occupe une place centrale dans le corpus kondakovien. Ses voyages au mont Sinaï et dans les monastères du mont Athos lui procurèrent un accès privilégié aux sources mêmes de cet art ; un rôle non moins important fut joué par la collection d'anciennes icônes - en partie préiconoclastes - du savant évêque Porfirij (Uspenskij), ami de Kondakov. La connaissance de ces sources permit à Kondakov d'avancer des hypothèses convaincantes concernant les origines de l'icône, notamment dans le portrait funéraire égyptien. Mais, tout en remontant à l'Antiquité, les icônes étaient peintes et vénérées en Russie, au XIX et au $\mathrm{xx}^{\mathrm{e}}$ siècles, de la même façon qu'au Moyen Âge. Cet art relevait donc aussi bien de l'histoire que de la modernité.

4 L'intérêt pour l'étude des icônes apparut en Occident pour d'autres raisons : la figure d'Adolphe-Napoléon Didron est exemplaire de ce mouvement. Plusieurs historiens et collectionneurs russes suivirent l'exemple de Didron : Ivan Saharov, Dimitrij Rovinskij, le comte Gregorij Stroganov et, enfin, le maître à penser de Kondakov, Fedor Buslaev. Tout comme Didron, ils s'appuyaient sur des sources particulières - les Guides ou manuels iconographiques pour les peintres d'icônes (en russe, ikonopisnyj podlinnik). Mais les auteurs russes avaient aussi un accès privilégié à l'art des icônes anciennes grâce aux milieux des vieux-croyants, conservateurs de cette tradition ancestrale.

5 Sur ce fond, les icônes apparurent dans les écrits de Kondakov d'abord à l'intérieur de ses ouvrages consacrés à d'autres sujets (ses voyages archéologiques, par exemple). En tant que membre de la Société pour la sauvegarde de l'icône russe, présidée par le comte Sergej Šeremetev, il développa ensuite un intérêt spécifique pour l'icône contemporaine. Enfin, il participa à la publication de la collection de Porfirij Uspenskij. Cet intérêt pour l'icône d'un universitaire russe s'insérant dans un mouvement plus large, celui de la redécouverte (et non pas l'invention, souligne Ivan Foletti) du sens esthétique de l'icône russe, mouvement représenté notamment par Pavel Muratov et auquel Matisse, lors de son séjour à Moscou en 1911, donna une résonnance internationale. Les deux ouvrages de Kondakov consacrés à l'iconographie de la Vierge (1911 et 1915, ce dernier en deux volumes), ainsi que son ouvrage dédié à l'icône, publié bien plus tard, vers la fin de sa vie (en russe à Prague et en anglais à Oxford, mais qu'Ivan Foletti cite dans une récente traduction française, parue en $2008 \mathrm{chez}$ Parkstone), apparaissaient donc liés à ce contexte. Dans son dernier ouvrage, Kondakov analysait la nature de l'icône à partir des données nouvelles, en réfléchissant sur sa place entre l'art et l'artisanat, entre la tradition enracinée dans l'Antiquité et préservée par le canon et sa sensibilité aux pénétrations extérieures.

6 La troisième partie du livre est consacrée aux problèmes méthodologiques, notamment à la relation de Kondakov avec la science occidentale (Kondakov, l'Occidente e le questioni di metodo). La base de la réflexion scientifique de Kondakov, affirme Foletti, provenait de l'Occident. Sa méthode qui fut celle d'un philologue objectiviste, à l'écriture télégraphique, axée sur l'étude de l'iconographie, sans pour autant oublier l'importance de la forme, empruntait aisément au vaste champ de la science contemporaine 
d'histoire de l'art. Si l'école de Vienne, et tout particulièrement la démarche d'Alois Riegl, ne semblait pas lui avoir servi de modèle, c'est avec celle de Gottfried Semper qu'il avait, selon Foletti, le plus d'affinité, notamment quant à la question du rapport entre le prototype et la copie. Mais l'influence déterminante, durant ses années de formation, fut néanmoins française, celle de Jules Labarte (1797-1880). Parmi les collaborateurs de ses années de maturité, les Français furent également nombreux: Kondakov entretenait des relations intenses avec Eugène Müntz, et devint un véritable maître à penser pour Charles Diehl et Gabriel Millet.

7 Ivan Foletti expose, ensuite, une fortune non moins importante de l'œuvre de Kondakov dans le monde anglo-saxon, en citant les exemples du byzantiniste anglais Ormonde Maddok Dalton, de l'Américain Thomas Whittemore, restaurateur de SainteSophie et créateur de l'Institut byzantin américain et, enfin, de Sir Ellis Hovell Minns, traducteur et éditeur des Russian Icons de Kondakov.

8 L'un des chapitres inédits dans la vie de Kondakov fut sa relation avec Antonio Muñoz, superintendant des biens culturels de la Rome fasciste, qui avait été byzantiniste au début de sa carrière et, sur le plan scientifique, devait beaucoup à Kondakov. Muñoz, connaissant personnellement le pape Pie XI qui était bibliophile, réussit à le convaincre de faire acheter par le Vatican le manuscrit du troisième volume de l'Iconographie de la Vierge; il obtint aussi, de Benito Mussolini, une bourse de 3000 livres par mois pour son ami. La nouvelle de cette bourse arriva à Prague un jour avant la mort du savant russe.

C'est ce manuscrit, ou plus exactement sa traduction française qui, presqu'au même moment, sort des presses de Viella, édité également par Ivan Foletti qui l'a retrouvé dans les archives du Vatican. La traduction fut réalisée, peu après l'achat du manuscrit, au sein de l'Institut pontifical oriental et préparée à la publication. Or, cette dernière ne se fit jamais, le manuscrit de Kondakov étant soumis aux critiques virulentes, notamment de l'un des plus importants médiévistes italiens de cette époque, Pietro Toesca. En effet, à la différence des deux premiers volumes de l'Iconographie de la Vierge, consacrés à l'art byzantin ou d'influence byzantine, qui gardent toute leur importance jusqu'à nos jours, ce troisième volume, qui traite de l'image de la Vierge dans l'art italien de la Renaissance, laisse perplexe quant à son intérêt en dehors de l'historiographie de l'histoire de l'art. Et même dans ce cas, sa publication serait sans doute mieux placée à la suite de celle des deux premiers volumes.

$\mathrm{Au}$ lecteur occidental, les publications d'Ivan Foletti ouvrent un nouveau domaine peu connu jusqu'ici, celui de l'historiographie de l'histoire de l'art en Russie, qui attend, comme l'auteur le souligne lui-même, des recherches et réflexions nouvelles. 